\title{
Multirobot rendezvous with bearing-only or range-only measurements
}

\author{
Ronghao Zheng ${ }^{*}$ and Dong Sun
}

\begin{abstract}
This paper studies distributed rendezvous strategies for multiple nonholonomic wheeled mobile robots with the aim of testing their practicality on real robots. We investigate control strategies which use just bearing-only or range-only measurements and do not need inter-robot radio communication to share the measurements. For the bearing-only case, two control laws proposed in our previous study are recalled and adapted. For the range-only case, rendezvous control laws for a two-robot system are proposed first and it is shown analytically a two-robot system achieves rendezvous globally under these control laws. Then the range-only-based control laws are extended to multirobot systems. Monte Carlo simulations indicate that a multirobot system achieves practical convergence under the range-only-based control laws. Experimental results illustrate the applicability and performance of the proposed control strategies for multiple wheeled-robot systems.
\end{abstract}

Keywords: Multirobot systems; Bearing-only measurement; Range-only measurement

\section{Background}

Recent theoretical and technological advances have spurred a broad interest to develop practical multirobot systems [1-5]. For mobile robots, navigation skill is one of their fundamental capabilities. Different navigation strategies are appropriate for different contexts. Different sensor types and sensing modules are used depending on the application scenarios. However, a problem of common interests and practical significance is how to perform tasks with less information and simpler sensors, such as using binary sensors, or using bearing-only or range-only sensors. This problem has attracted much attention in the multirobot community because an advantage of cooperation of teams of robots is that simple agents are able to perform complex tasks through mutual cooperation.

Bearing-only-based navigation is an approach to simplify the sensing system and is very useful in some cases. For example, for mobile robots equipped with single omnidirectional camera, or radar and sonar operating in passive listening mode, it is a practical requirement to design control strategies based only on measurements of bearings $[6,7]$.

*Correspondence: rzheng3-c@my.cityu.edu.hk

Department of Mechanical and Biomedical Engineering, City University of Hong Kong, 83 Tat Chee Avenue, Hong Kong 999077, China
Range-only-based navigation is another approach and it is useful especially in those scenarios when the robots can only sense the intensity of the signals emitted by other robots, or, for example, underwater applications often use acoustic equipments to measure ranges by registering the time of flight of an echo request and reply. Generally speaking, the control design and analysis become more challenging when each robot can only measure distances to other robots without bearing information. This approach has a lot of applications, such as localization and mapping $[8,9]$, formation control $[10,11]$, and target enclosing $[12,13]$. If a robot has knowledge of its location in a coordinate frame, then under some persistent excitation condition, the robot may be able to get an estimation of the positions of other robots. This idea has been exploited in works such as $[12,14]$. However, in this paper we are interested in those scenarios where there is no global localization system so that absolute position information is not available.

Recently, a lot of researchers have studied the use of range-only-based technique to address the target tracking problem. This task becomes more challenging when wheeled mobile robots are used. Because of the nonholonomic constraints, wheeled mobile robots have restrictions in mobility and typically cannot be controlled

\section{是 Springer}

(C) 2014 Zheng and Sun; licensee Springer. This is an Open Access article distributed under the terms of the Creative Commons Attribution License (http://creativecommons.org/licenses/by/4.0), which permits unrestricted use, distribution, and reproduction in any medium, provided the original work is properly credited. 
by linear controllers. For example, recently, Matveev et al. [15] proposed a sliding mode control law to drive wheeled mobile robots towards a target and circumnavigate the target at a predefined distance. In [16], a two-phase switched logic-based control strategy was proposed.

Inspired by the research on bearing-only and range-only navigation, in this paper we consider another common task, i.e., the rendezvous problem in which multiple nonholonomic mobile robots are required to meet at a single point (see $[17,18]$ and references therein). Rendezvous control is very useful in a variety of applications for multirobot systems. For example, a group of robots can be deployed to collect samples from a region, and after the task is finished, they are required to get together so that we can collect them and transport them to a new place. However, most existing rendezvous strategies require that every robot knows both bearing and range of its neighboring robots, which restricts many practical applications. In this paper, we study distributed rendezvous strategies using just either bearing-only or range-only measurements. The bearing-only-based control schemes are adapted from our previous work [19] and experimentally validated in this paper. Range-only-based control schemes are also developed. While target tracking often assumes that the pursuer is more maneuverable than the target, in the rendezvous application all robots employ a same control strategy and have same maneuver. This makes the control more difficult especially when only range information is available. The proposed control schemes are then experimentally implemented and validated on a group of wheeled mobile robots.

The work presented in this paper is an extension of our previous work reported in a conference version [20]. Although using a similar idea, the range-only controllers in this paper are redesigned. The first difference is, in [20], that to prove the convergence of the distance between the robot pair, the forward velocity assumes an infinite bound. However, in this paper, a more practical design is provided, which allows both forward and angular velocities to lie in bounded intervals. The second difference is that the convergence of the multirobot system under the range-only controllers is verified by using Monte Carlo simulations. The third difference is that the experimental platform is improved and a behavior-based collision avoidance algorithm is used, which allows us to use more robots in our experiments and allows us to test the performance of the proposed controllers in more realistic scenarios.

The rest of the paper is structured as follows. In 'Methods' section, 'Problem description' subsection introduces the problem studied in this paper; 'Bearing-only controllers' subsection presents two bearing-only-based control schemes adapted from our previous work;
'Range-only controllers' subsection proposes two rangeonly-based control schemes and proves their global stability for the two-robot case. These two control schemes are then naturally extended to the multirobot case. In 'Results and discussion' section, Monte Carlo simulations are then carried out to test the convergence of the range-onlybased control schemes in multirobot systems. 'Results and discussion' section also discusses the experimental detail and results under the proposed control schemes. Concluding remarks are given in 'Conclusions' section.

\section{Methods}

\section{Problem description}

Assume $N$-wheeled mobile robots moving in a 2D obstacle-free workspace. In this paper, we model each robot as a kinematic unicycle. Then the posture of the $i$ th robot is represented by a triple $\left(x_{i}, y_{i}, \theta_{i}\right)$ where $\left(x_{i}, y_{i}\right)$ specifies the Cartesian coordinate of the center of the robot's body and $\theta_{i}$ gives its orientation. The motion of each robot $i \in\{1,2, \ldots, N\}$ is governed by

$$
\begin{aligned}
& \dot{x}_{i}=v_{i} \cos \theta_{i}, \\
& \dot{y}_{i}=v_{i} \sin \theta_{i}, \\
& \dot{\theta}_{i}=\omega_{i} .
\end{aligned}
$$

Here, control signals $v_{i} \in\left[-v_{\max }, v_{\max }\right]$ and $\omega_{i} \in$ $\left[-\omega_{\max }, \omega_{\max }\right]$ are robot $i$ 's forward and angular speeds, respectively, and $v_{\max }$ and $\omega_{\max }$ (both positive) are bounds for forward and angular speeds, respectively. From (1), it can be seen that the mobile robot is subjected to the nonholonomic constraint $\dot{y}_{i} \cos \theta_{i}-\dot{x}_{i} \sin \theta_{i}=0$.

Our goal is to design distributed control schemes $\left(v_{i}, \omega_{i}\right)$ to get all the $N$ nonholonomic robots to congregate at a common location. With the distributed architecture, the controller of robot $i$ only uses locally measurable information without a common reference frame, i.e., global position information is unavailable. In this paper, we consider two types of measurements. The first one is bearing-only measurement, i.e., each robot can only measure the bearing angles of the detectable robots in its local frame. The second is range-only measurement, i.e., each robot is able to measure only its distances from other robots that it can detect.

In this paper, we assume that the interaction between robots is bidirectional, i.e., if robot $i$ can detect robot $j$ then it can also be detected by robot $j$ and we say that robots $i$ and $j$ are neighbors. This assumption is reasonable, for example, in the case where all the robots use omnidirectional sensors with identical parameters. We then represent the bidirectional interaction topology among robots with an undirected graph $\mathcal{G}=(V, E)$ where $V$ is the node set with each node corresponding to each robot and $E$ is the edge set such that $(i, j) \in E$ implies that robots $i$ and $j$ are neighbors. We denote the set of robot 
$i$ 's neighbors as $\mathcal{N}_{i}$, i.e., $\mathcal{N}_{i}=\{j \mid(j, i) \in E\}$. A graph $\mathcal{G}$ is said to be connected if there is a path between every distinct nodes. If for any two distinct nodes $i$ and $j$ there is an edge connecting them, $\mathcal{G}$ is said to be completely connected.

\section{Bearing-only controllers}

In this subsection, we recall two bearing-only control schemes published in our previous work [19]. These two bearing-only control schemes are slightly modified here in order to take the speed bounds into account. The proofs of global convergence can be found in the original paper. We aim to examine their practical performances in this paper. We let $\rho_{i j}:=\sqrt{\left(x_{i}-x_{j}\right)^{2}+\left(y_{i}-y_{j}\right)^{2}}$ denote the distance between robots $i$ and $j$. For each robot $i$, we define the anticlockwise angle difference between robot $i$ 's heading and the line-of-sight that would take it directly toward another robot $j$ as the bearing angle of robot $j$ in robot $i$ 's coordinate frame and denote it by $\alpha_{i j}$ (see Figure 1 ). To guarantee that $\alpha_{i j}$ is well-defined, it is assumed that for two distinct robots $i$ and $j, \rho_{i j}>0$ for all $t \geq 0$. If $\rho_{i j} \leq \varepsilon$, where $\varepsilon$ is an arbitrary small positive number, then robots $i$ and $j$ achieve rendezvous and merge to a single robot, either $i$ or $j$. Here, $\varepsilon$ can be considered as a measure of the physical size of the robot.

We adapt the following control scheme from [19]. For each robot $i$,

Controller 1:

$$
\left[\begin{array}{c}
v_{i} \\
\omega_{i}
\end{array}\right]= \begin{cases}\frac{\sum_{j \in \mathcal{N}_{i}}}{\left|\mathcal{N}_{i}\right|}\left[\begin{array}{c}
v_{\max } \cos \alpha_{i j} \\
\omega_{\max } \sin \alpha_{i j}
\end{array}\right], & \text { if } \mathcal{N}_{i} \neq \emptyset \\
{[0,0]^{T},} & \text { otherwise. }\end{cases}
$$

Here $\left|\mathcal{N}_{i}\right|$ denotes the cardinality of the neighbor set $\mathcal{N}_{i}$. When $\mathcal{G}$ is connected, we can see that $\left|\mathcal{N}_{i}\right| \geq 1$ for all $i$.

The convergence of a $N$-robot system under Controller 1 can be proved by using a Lyapunov-based method and is formally stated by the following result.

Theorem 1 ([19], Theorem 1). A system of $N$ mobile robots described by (1) rendezvous under Controller 1 provided that $\mathcal{G}$ is connected. Moreover, the energy function $V:=\frac{1}{2} \sum_{i=1}^{N} \sum_{j \in \mathcal{N}_{i}} \rho_{i j}^{2}$ keeps decreasing until the robots achieve rendezvous.

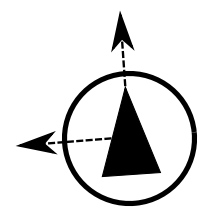

Robot $j$

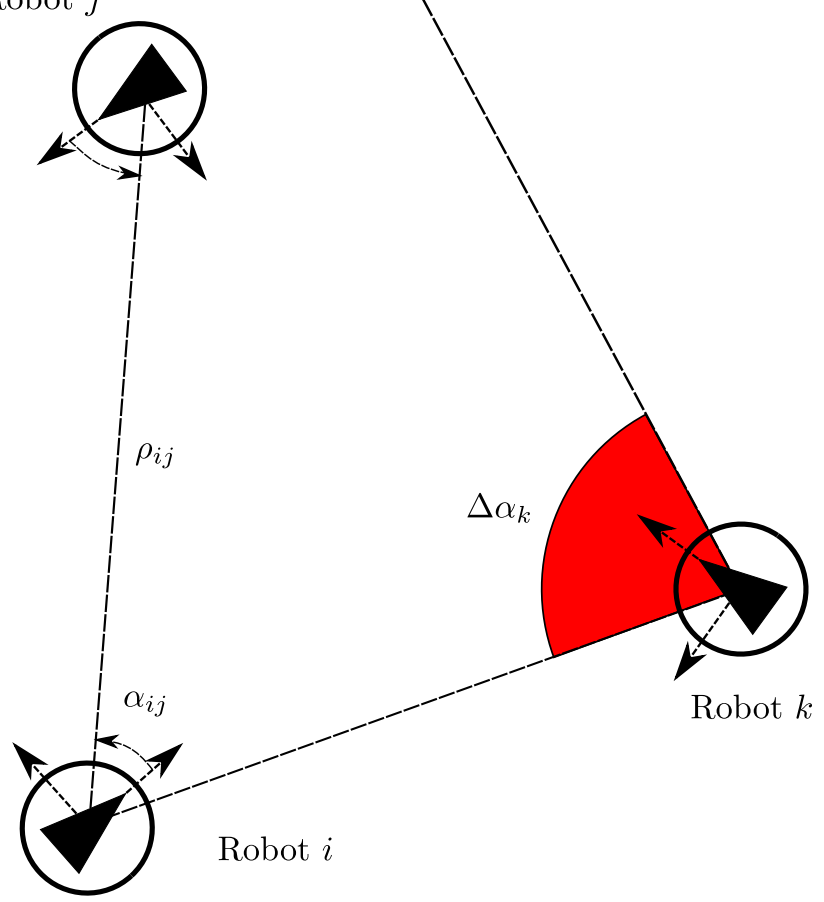

Figure 1 An illustration of $\rho_{i j}, \alpha_{i j}$ and the smallest circular sector of robot $k$. For robot $k_{,} \alpha_{k+}$ and $\alpha_{k-}$ correspond to $\alpha_{k i}$ and $\alpha_{k l}$ in this figure. 
When the interaction topology $\mathcal{G}$ between robots are completely connected, another bearing-only control scheme is proposed in [19] and adapted here. For each robot $i$,

Controller 2:

$$
\left[\begin{array}{c}
v_{i} \\
\omega_{i}
\end{array}\right]= \begin{cases}\frac{1}{2}\left[\begin{array}{c}
v_{\max }\left(\cos \alpha_{i+}+\cos \alpha_{i-}\right) \\
\omega_{\max }\left(\sin \alpha_{i+}+\sin \alpha_{i-}\right)
\end{array}\right], & \text { if } \Delta \alpha_{i} \in[0, \pi], \\
{[0,0]^{T},} & \text { otherwise. }\end{cases}
$$

Here, $\Delta \alpha_{i} \in[0,2 \pi)$ is defined to be the central angle of the smallest circular sector of robot $i$ which contains all the vectors $\left(\cos \alpha_{i j}\right.$, $\left.\sin \alpha_{i j}\right)$ for $j \in \mathcal{N}_{i}$, and $\alpha_{i+}$ and $\alpha_{i-}$ are defined to the bearing angles which correspond to the two radii enclosing robot $i$ 's circular sector (see Figure 1).

The convergence of a $N$-robot system under Controller 2 is formally stated by the following theorem.

Theorem 2 ([19], Theorem 4). A system of $N$ mobile robots described by (1) rendezvous under Controller 2 provided that $\mathcal{G}$ is completely connected, and the perimeter of the convex hull defined by the positions of robots keeps decreasing until the robots achieve rendezvous.

The idea behind Controller 2 can be explained as follows. When $\Delta \alpha_{i} \leq \pi$, robot $i$ is located at a vertex or on an edge of the convex hull defined by the positions of all robots. In that case, a robot tries to shorten the distances to its neighbors which are also at the vertices or on the edge; otherwise, it just keeps stationary. In this way, the perimeter of the convex hull will shrink to a point and the robots can meet each other at that point.

Remark 1. Using the pseudo-linearization technique, it can be proven that under Controllers 1 and 2, the meeting point is located in a bounded region which is determined by the robots' initial postures [19].

\section{Range-only controllers}

In this subsection, we propose two range-only control schemes to drive wheeled mobile robots to rendezvous. We first investigate the two-robot case and prove its global convergence. The control schemes are then generalized to deal with the $N$-robot case.

To facilitate the analysis, we consider the relative coordinates $\rho_{12}, \alpha_{12}$, and $\alpha_{21}$ between two robots 1 and 2 . After some algebraic manipulation, we get the following equations:

$$
\begin{aligned}
& \dot{\rho}_{12}=-v_{1} \cos \alpha_{12}-v_{2} \cos \alpha_{21}, \\
& \dot{\alpha}_{12}=-\omega_{1}+\frac{v_{1} \sin \alpha_{12}+v_{2} \sin \alpha_{21}}{\rho_{12}}, \\
& \dot{\alpha}_{21}=-\omega_{2}+\frac{v_{1} \sin \alpha_{12}+v_{2} \sin \alpha_{21}}{\rho_{12}} .
\end{aligned}
$$

Note that (4) is valid when $\rho_{12} \neq 0$.

Both robots are assumed to have access to only the current distance $\rho_{12}(t)$ and calculate its derivative $\dot{\rho}_{12}(t)$ by using a memory unit. The bearing information, i.e., $\alpha_{12}$ and $\alpha_{21}$, is not available. In the following, we propose two control schemes based on $\rho_{12}(t)$ and $\dot{\rho}_{12}(t)$, which drive two robots to rendezvous, i.e., $\rho_{12}(t) \rightarrow 0$ as $t \rightarrow+\infty$.

The first range-only control scheme we propose is Controller 3:

$$
\begin{aligned}
v_{i} & =k_{\nu} \chi\left\{\rho(t), \rho_{\nu}\right\}, \\
\omega_{i} & = \begin{cases}k_{\omega} \chi\left\{\rho(t), \rho_{\omega}\right\} \omega_{s} / \rho(t), & \text { if } \dot{\rho}(t)<0, \\
k_{\omega} \chi\left\{\rho(t), \rho_{\omega}\right\} \omega_{f} / \rho(t), & \text { if } \dot{\rho}(t) \geq 0,\end{cases}
\end{aligned}
$$

with $\omega_{f}>\omega_{s}>0$. Here $\chi(x, \bar{x})$ is a saturation function

$$
\chi(x, \bar{x}):= \begin{cases}x, & \text { if } x \leq \bar{x} \\ \bar{x}, & \text { if } x>\bar{x}\end{cases}
$$

In our case, the parameter $x$ of $f(x, \bar{x})$ is nonnegative and $\bar{x}$ is positive.

The rationale of Controller 3 can be explained by observing that when a robot finds itself approaching another robot, i.e., it is moving in the right direction, it rotates slowly (at the speed $\omega_{s}$ ) to try to keep on that direction as long as possible; otherwise, it rotates fast (at the speed $\left.\omega_{f}\right)$ to try to get to the right direction.

Under (5), $v_{1}=v_{2}:=v$ and $\omega_{1}=\omega_{2}:=\omega$. Define $\rho:=\rho_{12}, \alpha:=\left(\alpha_{12}+\alpha_{21}\right) / 2$ and $\beta:=\left(\alpha_{12}-\alpha_{21}\right) / 2$. Then

(4) can be rewritten as

$$
\begin{aligned}
& \dot{\rho}=-2 v \cos \alpha \cos \beta, \\
& \dot{\alpha}=-\omega+\frac{2 v \sin \alpha \cos \beta}{\rho}, \\
& \dot{\beta}=0 .
\end{aligned}
$$

From (6c), we can see that $\beta$ is a constant under Controller 3.

\section{Theorem 3. Design Controller 3 such that}

- $k_{v} \rho_{v} \leq v_{\max }, \rho_{v}=\rho_{\omega}$, and

- $k_{\omega} \omega_{f} \leq \omega_{\max }, \omega_{f}>\omega_{s}>2 k_{v} / k_{\omega}$.

A two-robot system described by (1) rendezvous under Controller 3 provided $\cos \beta \neq 0$.

Proof. It is clear that when $k_{v} \rho_{v} \leq v_{\max }$ and $k_{\omega} \omega_{f} \leq$ $\omega_{\max }$, both $v$ and $\omega$ are in their admissible intervals.

Since $\rho_{v}=\rho_{\omega}$ and $\omega_{f}>\omega_{s}>2 k_{v} / k_{\omega}$, then according to (5a) we have $\omega>2 v \sin \alpha \cos \beta / \rho$. Hence, $\dot{\alpha}<0$ in (6b). Define $t_{k}$ to be the instant when $\alpha\left(t_{k}\right)=-2 k \pi+\pi / 2$ for some integer $k$. 
(i) Case I: $\cos \beta>0$. According to (6),

$$
\begin{aligned}
\int_{\rho\left(t_{k}\right)}^{\rho\left(t_{k+1}\right)} \frac{d \rho}{\rho}= & \int_{-2 k \pi+\pi / 2}^{-2 k \pi-3 \pi / 2} \frac{2 v \cos \alpha \cos \beta}{\rho \omega-2 v \sin \alpha \cos \beta} d \alpha \\
= & -\int_{-2 k \pi-3 \pi / 2}^{-2 k \pi+\pi / 2} \frac{2 v \cos \alpha \cos \beta}{\rho \omega-2 v \sin \alpha \cos \beta} d \alpha \\
= & -\int_{-2 k \pi-3 \pi / 2}^{-2 k \pi-\pi / 2} \frac{\cos \alpha \cos \beta}{\frac{\rho \omega}{2 v}-\sin \alpha \cos \beta} d \alpha \\
& -\int_{-2 k \pi-\pi / 2}^{-2 k \pi+\pi / 2} \frac{\cos \alpha \cos \beta}{\frac{\rho \omega}{2 v}-\sin \alpha \cos \beta} d \alpha
\end{aligned}
$$

Because $v \geq 0$, when $\dot{\rho}(t)<0$, we have $\cos \alpha>0$, i.e., $\alpha \in(-2 k \pi-\pi / 2,-2 k \pi+\pi / 2)$. Similarly, when $\dot{\rho}(t) \geq 0$, we have $\cos \alpha \leq 0$, i.e., $\alpha \in[-2 k \pi-3 \pi / 2,-2 k \pi-\pi / 2]$. Hence,

$$
\begin{aligned}
\int_{\rho\left(t_{k}\right)}^{\rho\left(t_{k+1}\right)} \frac{d \rho}{\rho}= & -\int_{-3 \pi / 2}^{-\pi / 2} \frac{\cos \alpha \cos \beta d \alpha}{\frac{k_{\omega} \omega_{f}}{2 k_{v}}-\sin \alpha \cos \beta} \\
& -\int_{-\pi / 2}^{\pi / 2} \frac{\cos \alpha \cos \beta d \alpha}{\frac{k_{\omega} \omega_{s}}{2 k_{v}}-\sin \alpha \cos \beta} \\
= & \left.\ln \left(\frac{k_{\omega} \omega_{f}}{2 k_{v}}-\sin \alpha \cos \beta\right)\right|_{\alpha=-3 \pi / 2} ^{-\pi / 2} \\
& +\left.\ln \left(\frac{k_{\omega} \omega_{s}}{2 k_{v}}-\sin \alpha \cos \beta\right)\right|_{\alpha=-\pi / 2} ^{\pi / 2} \\
= & \ln \frac{\left(\frac{k_{\omega} \omega_{f}}{2 k_{v}}+\cos \beta\right)\left(\frac{k_{\omega} \omega_{s}}{2 k_{v}}-\cos \beta\right)}{\left(\frac{k_{\omega} \omega_{f}}{2 k_{v}}-\cos \beta\right)\left(\frac{k_{\omega} \omega_{s}}{2 k_{v}}+\cos \beta\right)}
\end{aligned}
$$

Because $0<\left(\frac{k_{\omega} \omega_{f}}{2 k_{v}}+\cos \beta\right)\left(\frac{k_{\omega} \omega_{s}}{2 k_{v}}-\cos \beta\right)<\left(\frac{k_{\omega} \omega_{f}}{2 k_{v}}-\right.$ $\cos \beta)\left(\frac{k_{\omega} \omega_{s}}{2 k_{v}}+\cos \beta\right)$, we get $\ln \rho\left(t_{k+1}\right)-\ln \rho\left(t_{k}\right)<0$, i.e., $\rho\left(t_{k+1}\right)<\rho\left(t_{k}\right)$; that is, $\rho(t)$ decreases in every period in which $\alpha(t)$ decreases by $2 \pi$. Because $\alpha(t)$ keeps decreasing, $\rho(t)$ will eventually approach 0 .

(ii) Case II: $\cos \beta<0$. The proof is similar to (i).

Using a similar idea as Controller 3 , the second rangeonly controller is proposed. In Controller 4, instead of applying a switch controller on the angular velocity $\omega_{i}$, we apply the switching control to the forward velocity $v_{i}$. Our second range-only controller is

\section{Controller 4:}

$$
\begin{aligned}
v_{i} & = \begin{cases}k_{s} \chi\left\{\rho(t), \rho_{v}\right\}, & \text { if } \dot{\rho}(t)<0, \\
k_{f} \chi\left\{\rho(t), \rho_{\nu}\right\}, & \text { if } \dot{\rho}(t) \geq 0,\end{cases} \\
\omega_{i} & =k_{\omega} \chi\left\{\rho(t), \rho_{\omega}\right\} \bar{\omega} / \rho(t),
\end{aligned}
$$

where $k_{f}>k_{s}>0$.

The convergence of $\rho$ under Controller 4 is stated in the following result.
Theorem 4. Design Controller 4 such that

- $k_{f} \rho_{v} \leq v_{\max }, \rho_{v}=\rho_{\omega}$, and

- $k_{\omega} \bar{\omega} \leq \omega_{\max }, k_{\omega} \bar{\omega}>2 k_{f}$.

A two-robot system described by (1) rendezvous under Controller 4 provided $\cos \beta \neq 0$.

Proof. The proof is similar to that of Theorem 3. Since $k_{\omega} \bar{\omega}>2 k_{f}$, thus $\dot{\alpha}<0$.

(i) Case I: $\cos \beta>0$. According to (6) and (7),

$$
\begin{aligned}
\int_{\rho\left(t_{k}\right)}^{\rho\left(t_{k+1}\right)} \frac{d \rho}{\rho}= & -\int_{-3 \pi / 2}^{-\pi / 2} \frac{\cos \alpha \cos \beta d \alpha}{\frac{k_{\omega} \bar{\omega}}{2 k_{s}}-\sin \alpha \cos \beta} \\
& -\int_{-\pi / 2}^{\pi / 2} \frac{\cos \alpha \cos \beta d \alpha}{\frac{k_{\omega} \bar{\omega}}{2 k_{f}}-\sin \alpha \cos \beta} \\
= & \left.\ln \left(\frac{k_{\omega} \bar{\omega}}{2 k_{s}}-\sin \alpha \cos \beta\right)\right|_{\alpha=-3 \pi / 2} ^{-\pi / 2} \\
& +\left.\ln \left(\frac{k_{\omega} \bar{\omega}}{2 k_{f}}-\sin \alpha \cos \beta\right)\right|_{\alpha=-\pi / 2} ^{\pi / 2} \\
= & \ln \frac{\left(\frac{k_{\omega} \bar{\omega}}{2 k_{s}}+\cos \beta\right)\left(\frac{k_{\omega} \bar{\omega}}{2 k_{f}}-\cos \beta\right)}{\left(\frac{k_{\omega} \bar{\omega}}{2 k_{s}}-\cos \beta\right)\left(\frac{k_{\omega} \bar{\omega}}{2 k_{f}}+\cos \beta\right)}<0
\end{aligned}
$$

thus, $\rho\left(t_{k+1}\right)<\rho\left(t_{k}\right)$.

(ii) Case II: $\cos \beta<0$. The proof is similar to (i).

To make Controllers 3 and 4 work, we need to avoid the case of $\cos \beta=0$. One way to do this is to introduce some random behavior, e.g., stop moving for a while, into the controllers if the robots detect that $\rho_{i j}$ keeps constant. The introduction of random behavior might also be helpful when the system suffers slow convergence, i.e., $|\cos \beta|$ is too small.

We generalize both Controllers 3 and 4 to the $N$-robot case. We call them Controllers $3 \mathrm{e}$ and $4 \mathrm{e}$ in this paper. To simplify the controller design of $\omega_{i}$, here we set $\rho_{\omega}=+\infty$.

Controller $3 e$ :

$$
\begin{aligned}
& v_{i}=k_{v} \chi\left[\sum_{j \in \mathcal{N}_{i}} \rho_{i j}, \rho_{v}\right], \\
& \omega_{i}= \begin{cases}k_{\omega} \omega_{s}, \quad \text { if } \sum_{j \in \mathcal{N}_{i}}\left(\rho_{i j} \dot{\rho}_{i j}\right)<0, \\
k_{\omega} \omega_{f}, \quad \text { if } \sum_{j \in \mathcal{N}_{i}}\left(\rho_{i j} \dot{\rho}_{i j}\right) \geq 0,\end{cases}
\end{aligned}
$$

where $\omega_{f}>\omega_{s}>0$. 


\section{Controller 4e:}

$$
\begin{aligned}
& v_{i}= \begin{cases}k_{s} \chi\left[\sum_{j \in \mathcal{N}_{i}} \rho_{i j}, \rho_{v}\right], & \text { if } \sum_{j \in \mathcal{N}_{i}}\left(\rho_{i j} \dot{\rho}_{i j}\right)<0, \\
k_{f} \chi\left[\sum_{j \in \mathcal{N}_{i}} \rho_{i j}, \rho_{v}\right], & \text { if } \sum_{j \in \mathcal{N}_{i}}\left(\rho_{i j} \dot{\rho}_{i j}\right) \geq 0,\end{cases} \\
& \omega_{i}=k_{\omega} \bar{\omega},
\end{aligned}
$$

where $k_{f}>k_{s}>0$.

Work is still ongoing to discover the convergence results for the $N$-robot case. However, in 'Results and discussion' section, it is shown by means of both Monte Carlo simulations and experiments that these two range-onlybased control schemes perform well provided that the interaction topology $\mathcal{G}$ among the robots is connected.

\section{Results and discussion} Monte Carlo simulations

The widely used Monte Carlo simulations were utilized to infer the convergence of the $N$-robot system under both Controllers $3 \mathrm{e}$ and $4 \mathrm{e}$ with connected interaction topology. The readers are referred to [21] for more details of Monte Carlo methods. In the simulations, for team size $N=3,4, \ldots, 10$, each $N$ is evaluated 100 times. All the initial configurations (positions and orientations) of the mobile robots and the control parameters for the controllers are generated randomly from uniform distributions over their domains respectively (see Table 1 ). Without loss of generality, we set $k_{v}=k_{\omega}=1$ in the simulations. In each evaluation, the initial positions of the

\section{Table 1 Parameters used in the Monte Carlo simulations}

\begin{tabular}{ll}
\hline Parameter & Value \\
\hline$N$ & $3, \ldots, 10$ \\
$k_{v}$ & 1 \\
$k_{\omega}$ & 1 \\
$\rho_{V}$ & $v_{\max }$ \\
$W$ & {$[10,100]$} \\
$x_{i}(0)$ & {$[0, W]$} \\
$y_{i}(0)$ & {$[0, W]$} \\
$\theta_{i}(0)$ & {$[0,2 \pi)$} \\
$d_{c}$ & $\sqrt{W^{2} / N}$ \\
$v_{\max }$ & {$[0.9,1.1]$} \\
$\omega_{\max }$ & {$[5,6]$} \\
$\omega_{s}$ & $\left(2 v_{\max }, 3\right]$ \\
$\omega_{f}$ & $\left(\omega_{s}+3, \omega_{\max }\right]$ \\
$\bar{\omega}$ & $\left(2 v_{\max }, 3\right]$ \\
$k_{s}$ & {$[0.4,0.5]$} \\
$k_{f}$ & {$[0.8,1.0]$} \\
\hline
\end{tabular}

robots $\left(x_{i}(0), y_{i}(0)\right)$ are chosen uniformly from a square with side $W$ and the initial orientations $\theta_{i}(0)$ are chosen from $[0,2 \pi)$ uniformly.

The topology $\mathcal{G}$ is generated in the following way: If the distance between a pair of robots is less than $d_{c}$, these two robots are connected to each other. We then test the connectivity of the resulting $\mathcal{G}$, if $\mathcal{G}$ is connected, e.g., the corresponding Laplacian has a rank of $N-1$, then we continue the evaluation; otherwise, this evaluation is abandoned and a new evaluation is carried out. To avoid using an over-connected $\mathcal{G}$, we chose $d_{c}=\sqrt{W^{2} / N}$ in the simulations. In each evaluation, we record the trajectories of the robots during 0 to $200 \mathrm{~s}$. The sampling time $T_{\mathrm{s}}$ is set to be $0.1 \mathrm{~s}$. It is tempting to chose a large $\omega_{\max }$, which causes no problem for a continuous-time system. However, a large $\omega_{\max }$ can cause a discrete-time realization of the system to be unstable. We found that keeping $\omega_{\max }<10 \times 2 \pi / T_{\mathrm{s}}$ provides enough stability margin.

The outcome of each evaluation is the normalized average distance $d(t)$ to the centroid of the robot team, i.e., $d(t)=\frac{\sum_{i=1}^{N}\left\|\left(x_{i}(t)-\bar{x}(t), y_{i}(t)-\bar{y}(t)\right)\right\|}{\sum_{i=1}^{N}\left\|\left(x_{i}(0)-\bar{x}(0), y_{i}(0)-\bar{y}(0)\right)\right\|}$, where $\bar{x}(t)=$ $\sum_{i=1}^{N} x_{i}(t) / N$ and $\bar{y}(t)=\sum_{i=1}^{N} y_{i}(t) / N$. We define the ratio of convergence as $\sigma=d(200 \mathrm{~s})$.

For the Controller $3 \mathrm{e}$, we recorded that the average ratio of convergence is $3.0561 \%$ and $\max \sigma=58.395 \%$. For the Controller $4 \mathrm{e}$, we recorded that the average ratio of convergence is $24.683 \%$ and $\max \sigma=83.808 \%$. Both results imply the Controllers $3 \mathrm{e}$ and $4 \mathrm{e}$ lead to practical convergence.

\section{Experimental platform}

The proposed control schemes were implemented and tested for the case of five e-puck robots [22] shown in Figure 2. The experiments were conducted in a lab

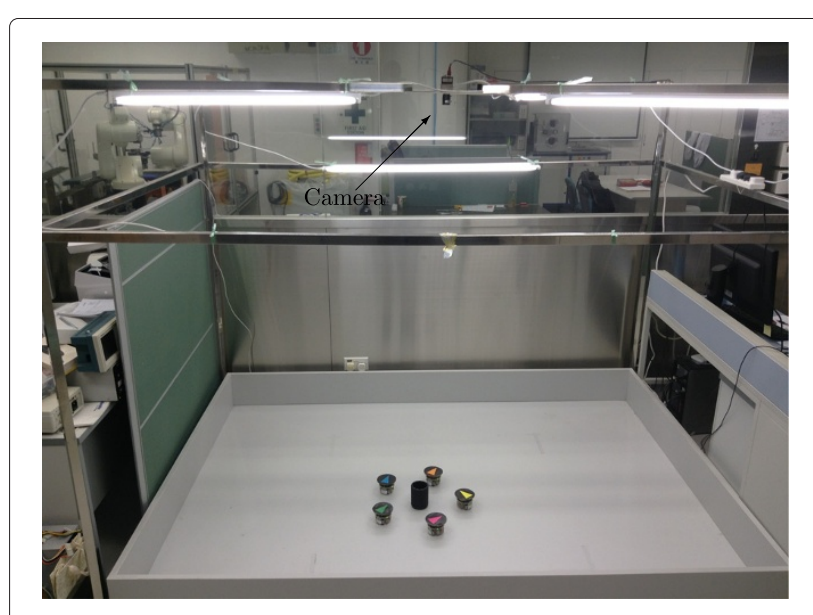

Figure 2 Experimental platform. 
environment. Because the sensing capability of the e-puck robot is very limited, in the current implementation, an overhead camera is used to track the absolute postures of the robots. The information from the tracking system is then used to produce the local bearing or range measurements, which are utilized in order to mimic local and distributed implementation of the proposed control schemes.

\section{Collision avoidance}

In the theoretical analysis, the robots are treated as moving points. However, real robot is never a point, and therefore collision avoidance cannot be negligible when several robots work together in a region. Collision avoidance itself, especially for nonholonomic mobile robots, is a challenging problem (see [23] and references therein). In our experiments, a behavior-based algorithm is adopted, which is straightforward and computationally effective.

It should be pointed out that using only bearing or range information is not enough to avoid collision. We need to assume that within a certain sensing zone $Q^{i}:=$ $\left\{j \mid \rho_{i j} \leq d_{s}, j \neq i\right\}$, a robot can detect both the bearing and range information, i.e., $\alpha_{i j}$ and $\rho_{i j}$, about other robots (see Figure 3). This can be done, for example, by using short-range infrared proximity sensors mounted around the body of the mobile robot. The forward speed $v_{i}$ is multiplied by a variable $m_{i}$ and become the new control signal

$$
\tilde{v}_{i}=m_{i} v_{i}
$$

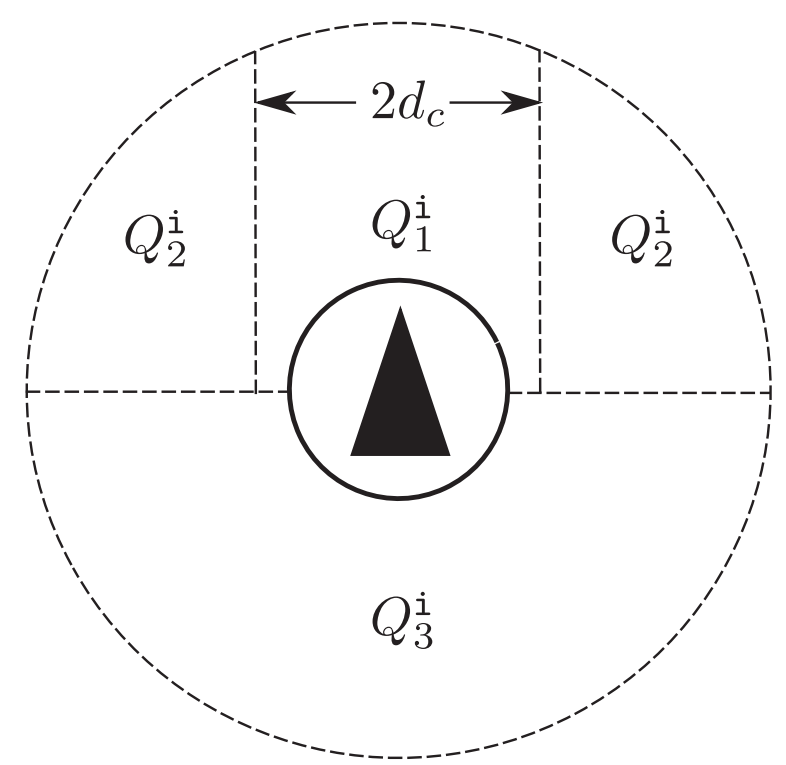

Figure 3 The partition of $Q_{1}^{i}, Q_{2}^{i}$, and $Q_{3}^{i}$ when $v_{i}>\mathbf{0}$. where

$$
m_{i}= \begin{cases}0, & \text { if } Q_{1}^{i} \neq \emptyset, \\ 0, & \text { if } Q_{2}^{i} \neq \emptyset \text { and }\left|v_{i}\right|<\left|v_{j}\right|, \exists j \in Q_{2}^{i}, \\ 2, & \text { if } Q_{1}^{i}=\emptyset, Q_{2}^{i} \neq \emptyset \text { and }\left|v_{i}\right|>\left|v_{j}\right|, \forall j \in Q_{2}^{i}, \\ 2, & \text { if } Q_{1}^{i}=\emptyset, Q_{2}^{i}=\emptyset, \text { and } Q_{3}^{i} \neq \emptyset .\end{cases}
$$

The partitions $Q_{1}^{i}, Q_{2}^{i}$, and $Q_{3}^{i}$ are formally defined as

$$
\begin{aligned}
& Q_{1}^{i}:=\left\{j\left|\cos \alpha_{i j} \cdot v_{i} \geq 0 \wedge \rho_{i j}\right| \sin \alpha_{i j} \mid \leq d_{c}\right\} \cap Q^{i}, \\
& Q_{2}^{i}:=\left\{j\left|\cos \alpha_{i j} \cdot v_{i} \geq 0 \wedge \rho_{i j}\right| \sin \alpha_{i j} \mid>d_{c}\right\} \cap Q^{i}, \\
& Q_{3}^{i}:=\left\{j \mid \cos \alpha_{i j} \cdot v_{i}<0\right\} \cap Q^{i} .
\end{aligned}
$$

Notice that $\tilde{v}_{i}$ may not be achieved due to saturation of the actuator.

Since the working area is bounded, the robots should also avoid collision with the boundaries and keep inside the working area. To do that, once detecting the boundaries, a robot stops moving forward and rotates at the maximal angular speed for a short time span until its forward direction (the direction of $v_{i}$ ) points into the bounded area again.

\section{Experimental results}

A variety of experiments are conducted using discretetime versions of the proposed controllers. The sampling time $T_{\mathrm{s}}$ is set to be $0.1 \mathrm{~s}$. The parameters used in the experiments are given in Table 2. In the experiments, the robots are considered to achieve rendezvous if there is a path connecting all the robots and each edge of the path is smaller than $d_{\text {min }}$. Despite the time delays caused by image processing and wireless communication and the inconsistent orientation values when the vision system may fail to identity the orientation of a robot

Table 2 Parameters used in the experiments

\begin{tabular}{ll}
\hline Parameter & Value \\
\hline$v_{\max }$ & $6 \mathrm{~cm} / \mathrm{s}$ \\
$\omega_{\max }$ & $2.43 \mathrm{rad} / \mathrm{s}$ \\
$d_{s}$ & $15 \mathrm{~cm}$ \\
$d_{c}$ & $9 \mathrm{~cm}$ \\
$d_{\min }$ & $12 \mathrm{~cm}$ \\
$k_{v}$ & 1 \\
$k_{\omega}$ & 1 \\
$\rho_{V}$ & $1.94 \mathrm{rad} / \mathrm{s}$ \\
$\omega_{s}$ & $1.46 \mathrm{rad} / \mathrm{s}$ \\
$\omega_{f}$ & $0.63 \mathrm{rad} / \mathrm{s}$ \\
$\bar{\omega}$ & 0.3 \\
$k_{s}$ & 1.0 \\
$k_{f}$ &
\end{tabular}


during a certain time period, the experimental outcome is very encouraging and indicates the robustness of the controllers.

\section{Controller 1}

Figure 4 shows the connected interaction topology $\mathcal{G}$ among the robots we used in our experiments.

Figure 5 shows the experimental result of the rendezvous under Controller 1 captured by the overhead camera. In Figure 5a, the initial postures of the robots are shown. In Figure 5b,c,d the actual trajectories of the robots, which are represented by sequences of colored dots, and their final postures at different time instants are also shown. Figure 6 confirms Theorem 1 that the energy function $V:=\frac{1}{2} \sum_{i=1}^{N} \sum_{j \in \mathcal{N}_{i}} \rho_{i j}^{2}$ decays all the time until the robots rendezvous.

In our program, the routines run periodically based on a Windows timer. Sometimes the routines may run out of the time slot allocated then the positions of the robots cannot be recorded. This explains the missing parts of the trajectories. In analysis, for example, calculating the evolution of the energy function, we recover the missing parts of the trajectories through linear interpolation.

\section{Controller 2}

Figure 7 shows the robots rendezvous under Controller 2. The perimeter $L$ of the convex polygon defined by the positions of robots is plotted as a function of time in Figure 8 . Figure 8 validates Theorem 2 that the perimeter decays all the time until the robots meet.

\section{Controller $3 e$}

To apply Controllers $3 \mathrm{e}$ and $4 \mathrm{e}, \dot{\rho}_{i j}(k)$ is approximated by $\left[\rho_{i j}(k)-\rho_{i j}(k-1)\right] / T_{\mathrm{s}}$ where $T_{\mathrm{s}}$ is the sampling period. The measurement error of $\rho_{i j}$ may introduce a rapid switching in the control signal. To prevent that, we use a simple filter $\rho_{i j}(k)=\sum_{l=0}^{n} a_{l} \hat{\rho}_{i j}(k-l)$ to reduce the measurement error effect. In our experiment, we choose $n=2$ and $a_{0}=0.8, a_{1}=a_{2}=0.1$.

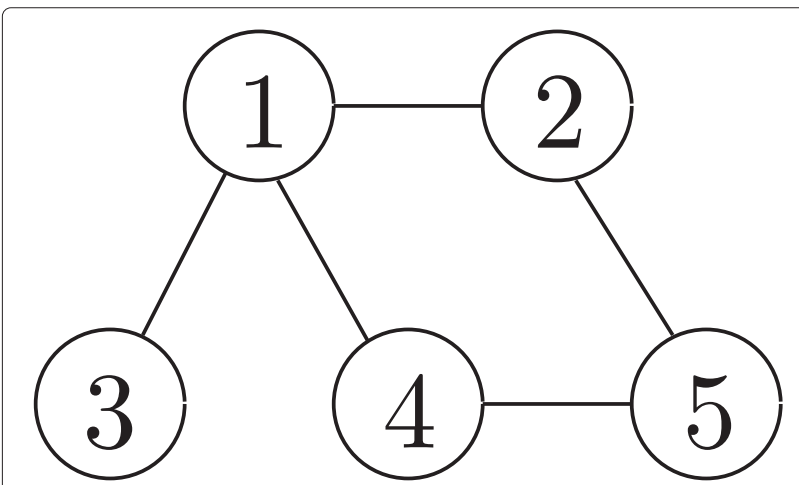

Figure 4 Interaction topology $\mathcal{G}$ among five robots.
We test the performance of Controller 3e under the connected topology of Figure 4. The result is presented in Figure 9. The evolution of the average distances to the group centroid is given in Figure 10.

\section{Controller $4 e$}

For the Controller 4e, we also use the interaction topology in Figure 4. The experimental result of rendezvous under Controller 4e is given in Figures 11 and 12.

It is not a surprising result that Controllers $3 \mathrm{e}$ and $4 \mathrm{e}$ take longer time to rendezvous than Controllers 1 and 2 do because Controllers $3 \mathrm{e}$ and $4 \mathrm{e}$ use only scalar information while Controllers 1 and 2 use vector information. By comparing the performances of Controllers $3 \mathrm{e}$ and $4 \mathrm{e}$, it can be seen that a switching control of the angular speed (Controller 3e) performs better than the one of the forward speed (Controller 4e). By comparing the experimental results, those shown in Figures 10 and 12 for example, we find that Controller $3 \mathrm{e}$ reduces the number of oscillations of $\rho_{i j}$ so it leads to faster convergence. This result also coincides with the observation from the Monte Carlo simulations.

\section{Conclusions}

In this paper, we study control schemes for driving a group of wheeled robots with nonholonomic constraints to a common location. The proposed control schemes use only the measurements of local bearing angles or only the measurements of distances among the robots. Our purpose is to examine whether the theoretical results obtained for bearing-only and range-only control schemes could be applied in practice to a real multirobot system. To this end, experiments are conducted on a team of e-puck robots. Given that there are unmodeled dynamic delays in the system due to sensing and information processing and the switching of controllers to deal with collision avoidance which are not accounted for in the theoretical analysis, the presented results are very positive.

However, these results are still preliminary. Further research will include developing a more sophisticated method of collision avoidance and implementing the proposed control schemes on more realistic scenarios, such as direct and dynamic interaction topology among mobile robots. Another research topic is to consider sensors with limited field-of-view and bounded range. We also plan to implement the proposed control schemes on mobile robots which have self-localization capability, such as those used in $[3,5]$, and develop the local localization technique for multirobot systems [24,25]. There are still many open questions in regards to the convergence of a $N$-robot system. Monte Carlo simulations indicate that Controllers $3 \mathrm{e}$ and $4 \mathrm{e}$ achieve practical convergence, but a formal proof of this assertion remains an open problem. 


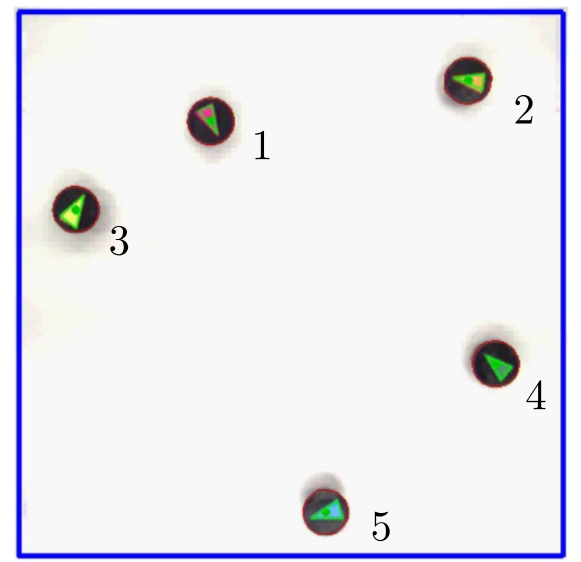

(a)

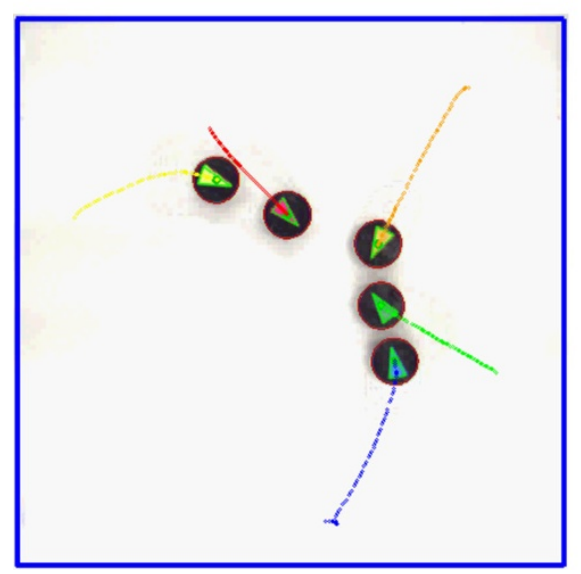

(c)

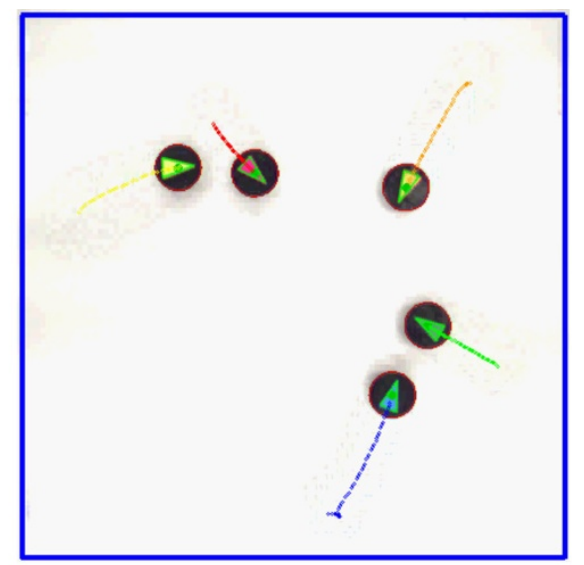

(b)

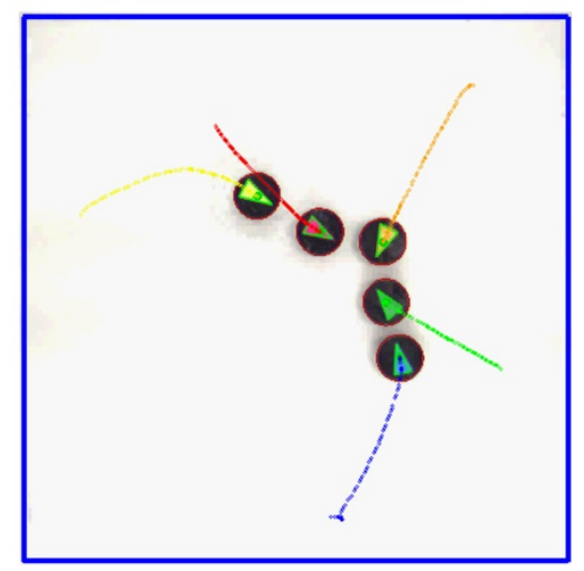

(d)

Figure 5 Rendezvous under Controller 1. (a) $t=0 \mathrm{~s}$. (b) $t=5 \mathrm{~s}$. (c) $t=10 \mathrm{~s}$. (d) $t=14 \mathrm{~s}$.

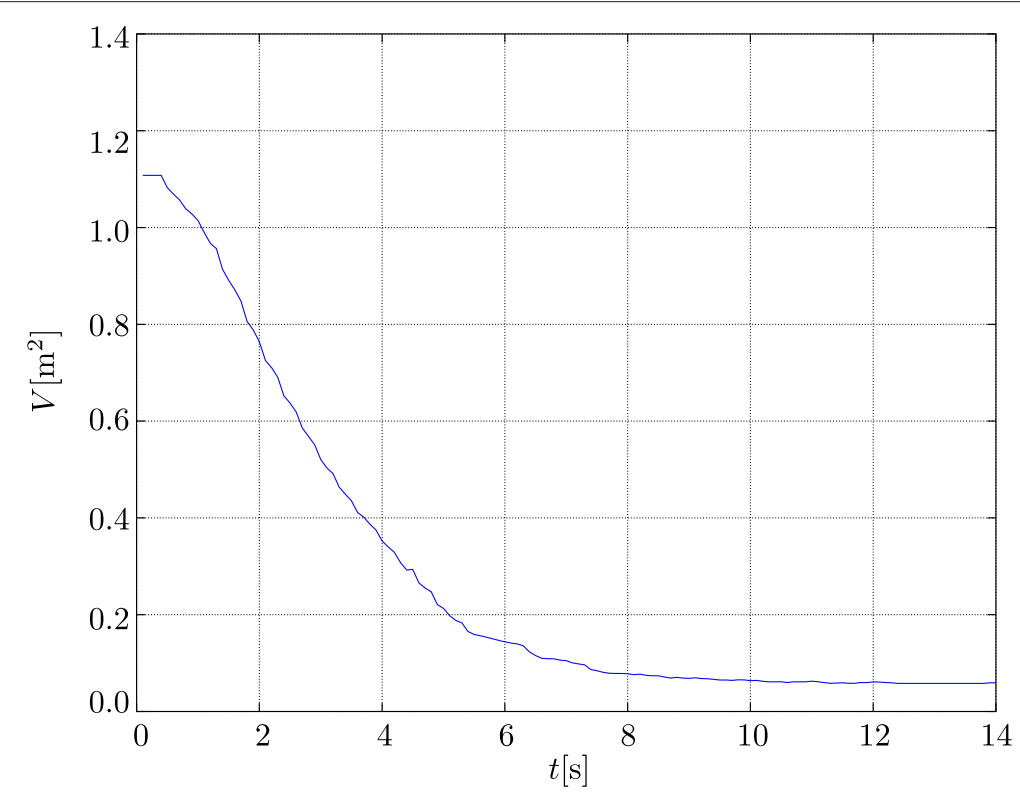

Figure 6 Evolution of the energy function $\boldsymbol{V}(\boldsymbol{t})$. Here $V(t):=\frac{1}{2} \sum_{i=1}^{N} \sum_{j \in \mathcal{N}_{i}} \rho_{i j}^{2}(t)$. 


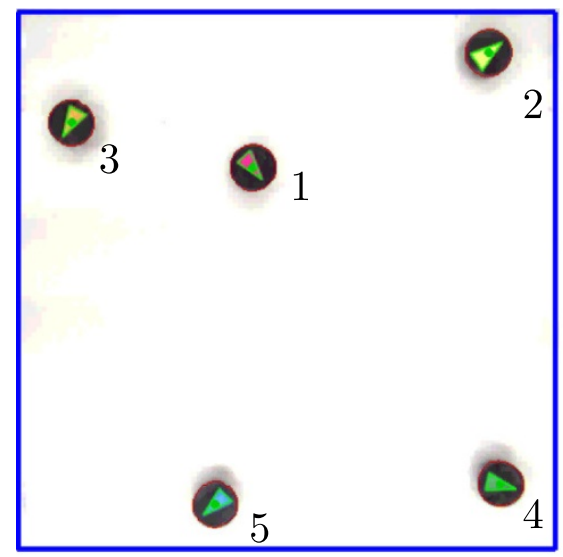

(a)

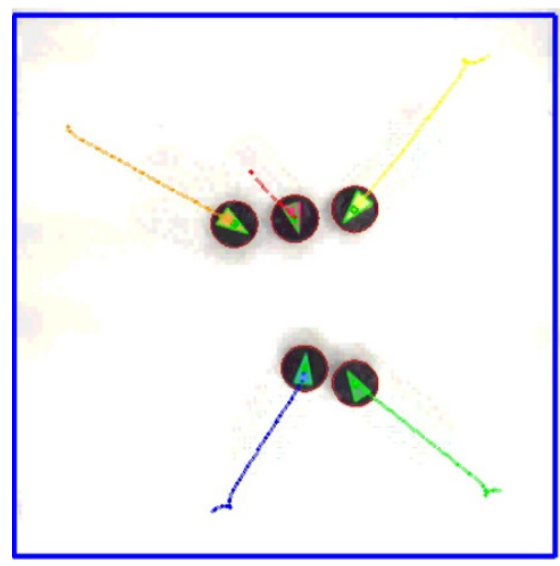

(c)

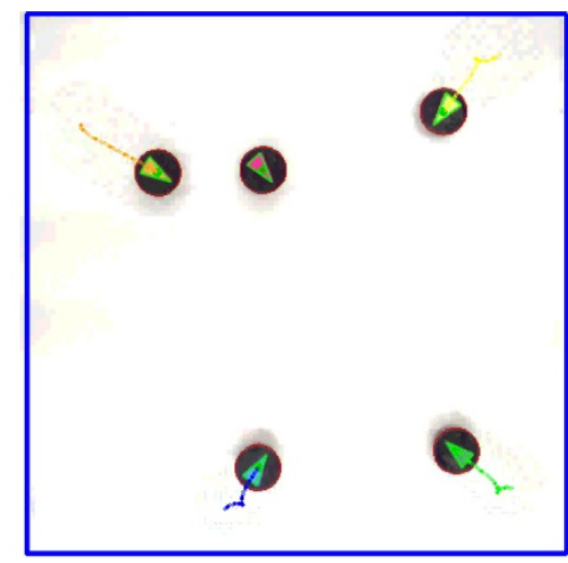

(b)

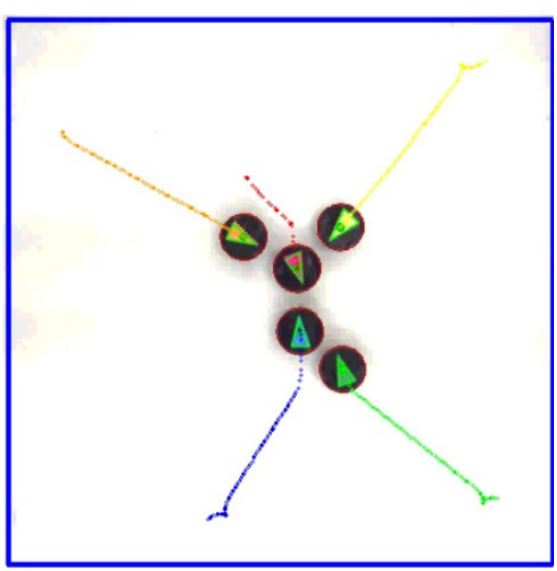

(d)

Figure 7 Rendezvous under Controller 2. (a) $t=0 \mathrm{~s}$. (b) $t=5 \mathrm{~s}$. (c) $t=11 \mathrm{~s}$. (d) $t=13 \mathrm{~s}$

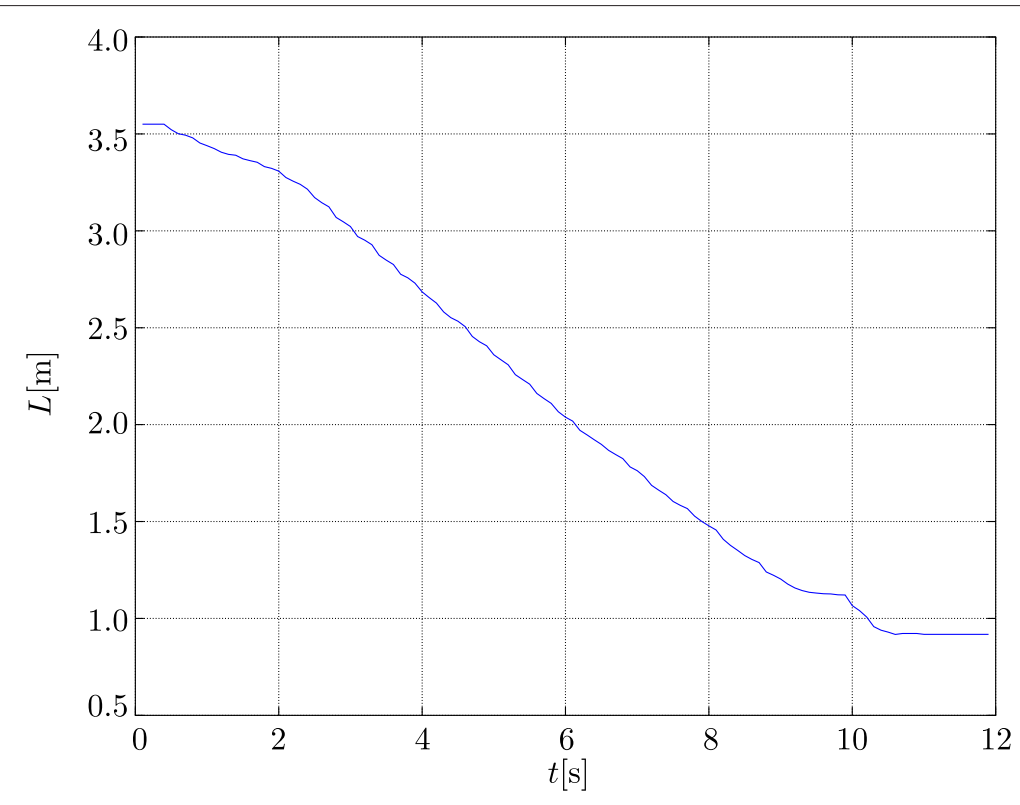

Figure 8 Evolution of the perimeter $L(t)$. 


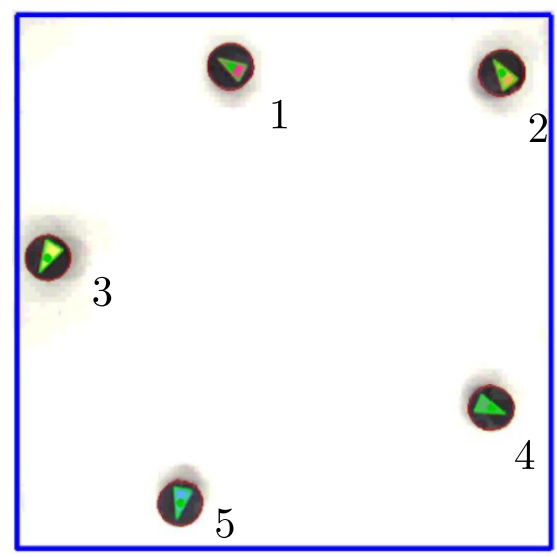

(a)

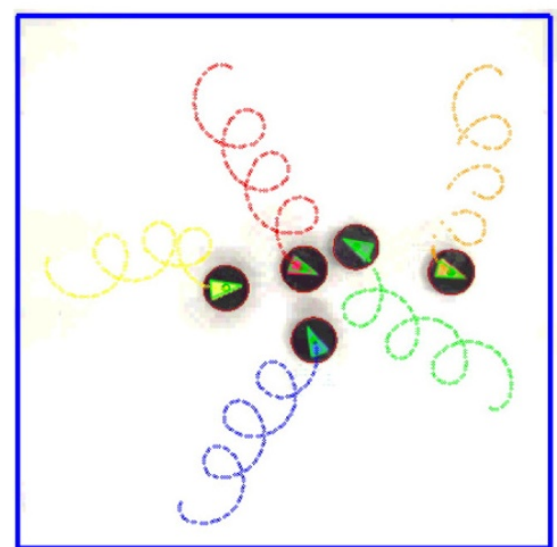

(c)

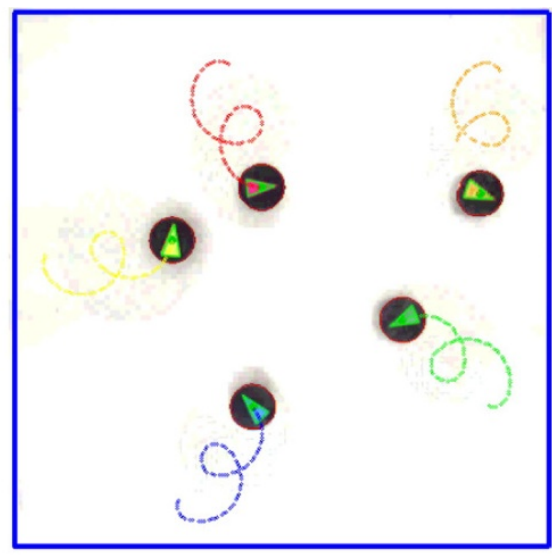

(b)

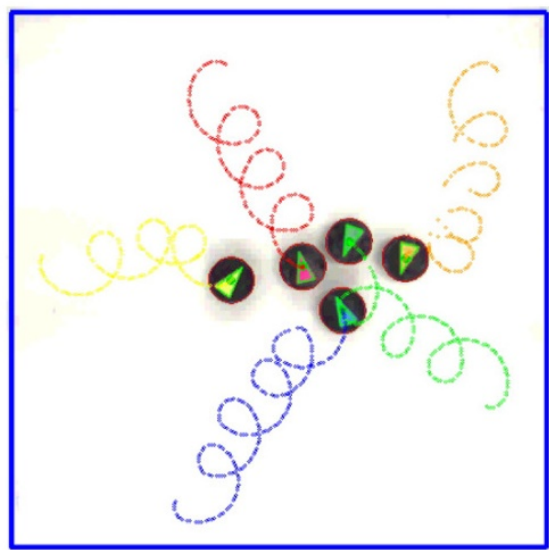

(d)

Figure 9 Rendezvous under Controller 3e. (a) $t=0 \mathrm{~s}$. (b) $t=5 \mathrm{~s}$. (c) $t=11 \mathrm{~s}$. (d) $t=13 \mathrm{~s}$.

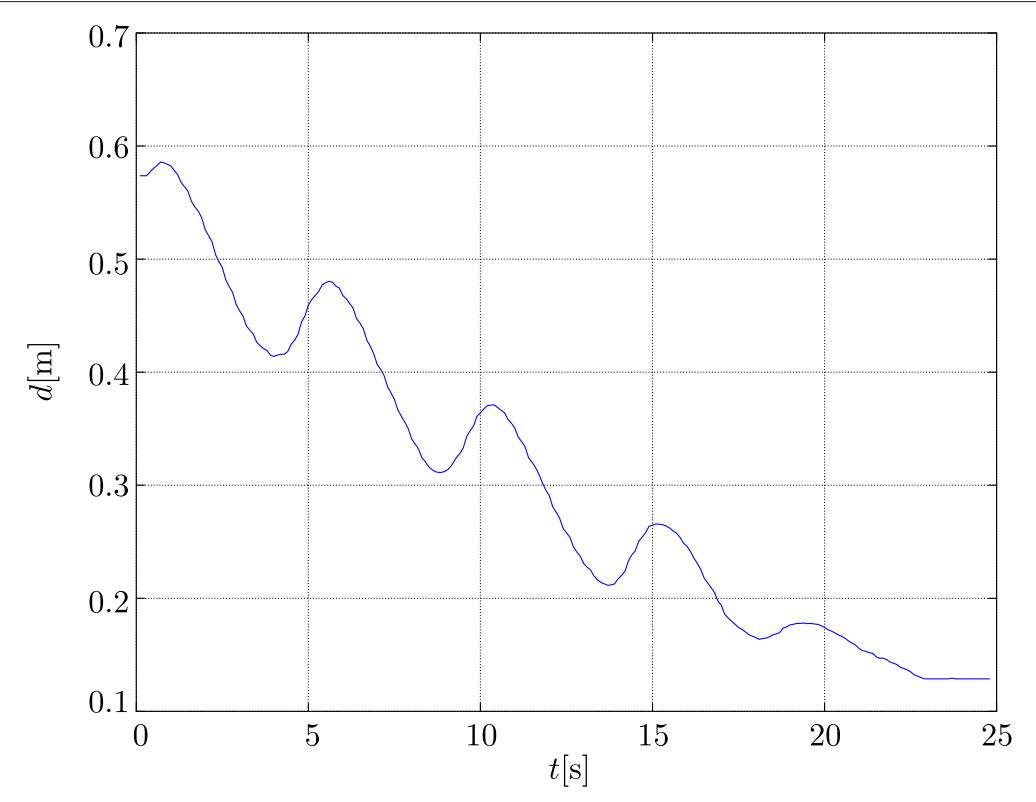

Figure 10 Evaluation of the average distance to group centroid $d(t)$. 


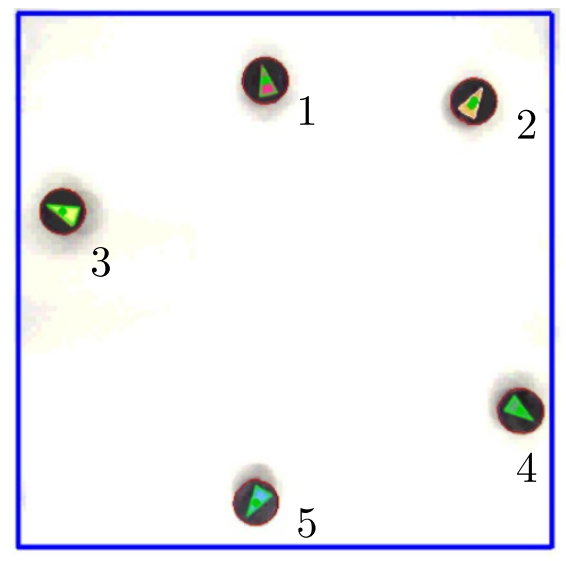

(a)

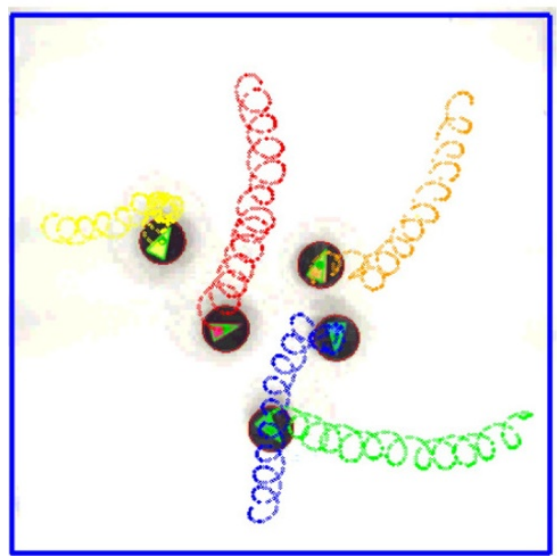

(c)

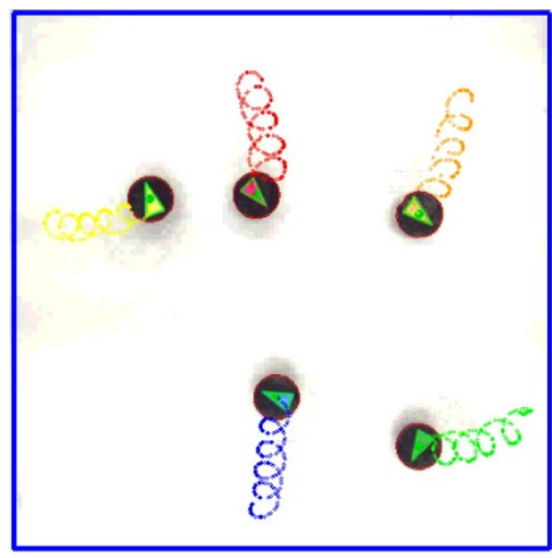

(b)

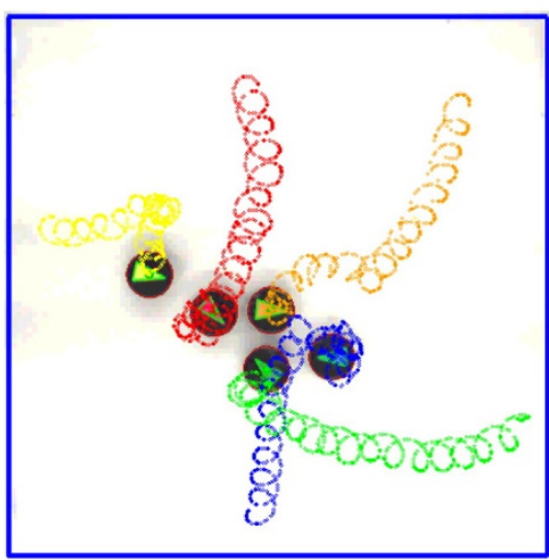

(d)

Figure 11 Rendezvous under Controller 4e. (a) $t=0 \mathrm{~s}$. (b) $t=20 \mathrm{~s}$. (c) $t=50 \mathrm{~s}$. (d) $t=73 \mathrm{~s}$.

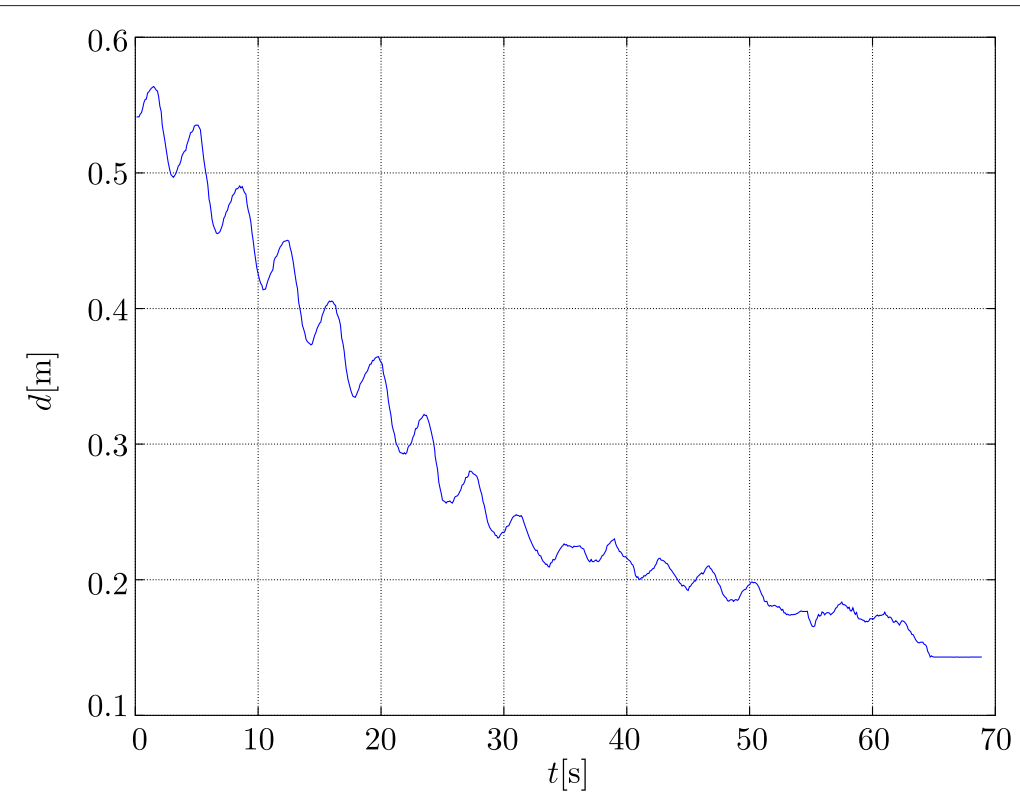

Figure 12 Evaluation of the average distance to group centroid $d(t)$. 


\section{Competing interests}

The authors declare that they have no competing interests.

\section{Authors' contributions}

RZ and DS designed the study, developed the methodology, collected the data, performed the analysis, and wrote the manuscript. Both authors read and approved the final manuscript.

\section{Acknowledgements}

The work of RZ is supported through the Hong Kong PhD Fellowship Scheme. The work is also supported by a grant from the Research Grants Council, Hong Kong Special Administrative Region, China, under Grant CityU 119612 and a Collaborative Research Fund with Project no. CUHK6/CRF/13G.

Received: 12 April 2014 Accepted: 5 August 2014

Published online: 01 October 2014

\section{References}

1. Marshall JA, Fung T, Broucke ME, D'Eleuterio GMT, Francis BA (2006) Experiments in multirobot coordination. Robot Autonomous Syst 54(3):265-275

2. Ren W, Chao H, Bourgeous W, Sorensen N, Chen YQ (2008) Experimental validation of consensus algorithms for multivehicle cooperative control. IEEE Trans Control Syst Technol 16(4):745-752

3. Sun D, Wang C, Shang W, Feng G (2009) A synchronization approach to trajectory tracking of multiple mobile robots while maintaining time-varying formations. IEEE Trans Robot 25(5):1074-1086

4. Yan X, Chen J, Sun D (2012) Multilevel-based topology design and shape control of robot swarms. Automatica 48(12):3122-3127

5. Li X, Sun D, Yang J (2013) A bounded controller for multirobot navigation while maintaining network connectivity in the presence of obstacles. Automatica 49(1):285-292

6. Farina A (1999) Target tracking with bearings-only measurements. Signal Process 78(1):61-78

7. Guo J, Yan G, Lin Z (2011) Balanced circular formation control based on gossip communication and angle of arrival information In: Proceedings of the 2011 Chinese Control Conference. Yantai, 22-24 July 2011, pp 6036-6041

8. Cahoon L, Hinich M (1976) A method for locating targets using range only. IEEE Trans Inf Theory 22(2):217-225

9. Kantor $\mathrm{G}$, Singh $\mathrm{S}$ (2002) Preliminary results in range-only localization and mapping In: Proceedings of 2002 IEEE International Conference on Robotics and Automation, Washington D.C., 11-15 May 2002, pp 1818-1823

10. Cao M, Yu C, Anderson B (2011) Formation control using range-only measurements. Automatica 47(4):776-781

11. Soares J, Aguiar A, Pascoal A, Martinoli A (2013) Joint ASV/AUV range-based formation control: theory and experimental results In: IEEE International Conference on Robotics and Automation, Karlsruhe, 6-10 May 2013, pp 5579-5585

12. Shames I, Dasgupta S, Fidan B, Anderson B (2012) Circumnavigation using distance measurements under slow drift. IEEE Trans Automatic Control 57(4):889-903

13. Cao Y, Muse J, Casbeer D, Kingston D (2013) Circumnavigation of an unknown target using UAVs with range and range rate measurements In: Proceedings of IEEE Conference on Decision and Control, Florence, 10-13 December 2013, pp 3617-3622

14. Bopardikar S, Bullo F, Hespanha J (2008) A pursuit game with range-only measurements In: Proceedings of IEEE Conference on Decision and Control, Cancun, 9-11 December 2008, pp 4233-4238

15. Matveev AS, Teimoori H, Savkin AV (2011) Range-only measurements based target following for wheeled mobile robots. Automatica 47(1):177-184

16. Namaki-Shoushtari O, Pedro Aguiar A, Khaki-Sedigh A (2012) Target tracking of autonomous robotic vehicles using range-only measurements: a switched logic-based control strategy. Int J Robust Nonlinear Control 22(17):1983-1998

17. Lin Z, Francis B, Maggiore M (2005) Necessary and sufficient graphical conditions for formation control of unicycles. IEEE Trans Automatic Control 50(1):121-127
18. Dimarogonas D, Kyriakopoulos K (2007) On the rendezvous problem for multiple nonholonomic agents. IEEE Trans Automatic Control 52(5):916-922

19. Zheng R, Sun D (2013) Rendezvous of unicycles: a bearings-only and perimeter shortening approach. Syst Control Lett 62(5):401-407

20. Zheng R, Sun D (2013) Rendezvous of wheeled mobile robots using bearings-only or range-only measurements In: Proceedings of IEEE International Conference on Robotics and Biomimetics, Shenzhen, 12-14 December 2013

21. Kalos M, Whitlock P (2008) Monte Carlo methods. Wiley-VCH, Weinheim

22. Mondada F (2014) e-puck education robot. http://www.e-puck.org/. Accessed 27 February 2014

23. Mastellone S, Stipanović D, Graunke C, Intlekofer KA, Spong M (2008) Formation control and collision avoidance for multi-agent non-holonomic systems: theory and experiments. Int J Robot Res 27(1):107-126

24. Chen H, Sun D, Yang J, Chen J (2010) Localization for multirobot formations in indoor environment. IEEE/ASME Trans Mechatronics 15(4):561-574

25. Chen H, Sun D, Yang J (2009) Global localization of multirobot formations using ceiling vision slam strategy. Mechatronics 19(5):617-628

\section{doi:10.1186/s40638-014-0004-5}

Cite this article as: Zheng and Sun: Multirobot rendezvous with bearing-only or range-only measurements. Robotics and Biomimetics 2014 1:4.

\section{Submit your manuscript to a SpringerOpen ${ }^{\circ}$ journal and benefit from:}

- Convenient online submission

- Rigorous peer review

- Immediate publication on acceptance

- Open access: articles freely available online

- High visibility within the field

- Retaining the copyright to your article

Submit your next manuscript at $>$ springeropen.com 